\title{
Antisense RNA transcripts in the blood may be novel diagnostic markers for colorectal cancer
}

\author{
TOMOHIRO KUROKAWA ${ }^{1}$, KEISUKE KOHNO ${ }^{1}$, KENTARO NAGAI ${ }^{1}$, MITSURU CHIBA $^{2}$, SUGIRU PAK ${ }^{1}$, \\ SOICHIRO MURATA ${ }^{1}$, KIYOSHI FUKUNAGA ${ }^{1}$, HIROSHI YASUE ${ }^{3}$ and NOBUHIRO OHKOHCHI ${ }^{1}$ \\ ${ }^{1}$ Department of Gastrointestinal and Hepato-Biliary-Pancreatic Surgery, Faculty of Medicine, \\ University of Tsukuba, Tsukuba, Ibaraki 305-8575; ${ }^{2}$ Department of Biomedical Sciences, Division of \\ Medical Life Sciences, Hirosaki University Graduate School of Health Sciences, Hirosaki, Aomori 036-8564; \\ ${ }^{3}$ Genome Resource Unit, National Institute of Agrobiological Sciences, Tsukuba, Ibaraki 305-0901, Japan
}

Received August 12, 2015; Accepted January 6, 2017

DOI: $10.3892 / 01.2017 .6572$

\begin{abstract}
Numerous genetic studies have been conducted regarding the occurrence of colorectal cancer (CRC) and the prognosis using microarrays. However, adequate investigations into the diagnostic application of microarrays have yet to be performed. The simplicity and accuracy of diagnosis and prognosis tracking are important requirements for its processes, and the use of blood cells for diagnosis is considered to be suitable to meet these requirements. The patients involved in the study were 28 preoperative patients with CRC and 6 healthy individuals who served as controls. RNA was extracted from the blood cells of the patients and analyzed using a sense/antisense RNA custom microarray. In the patients with CRC, the expression levels of 20 sense RNA and 20 antisense RNA species were identified as being significantly altered compared with that of the healthy volunteers $(\mathrm{P}<0.05$; fold-change, $>2.0)$. Cluster analysis of these RNA species revealed that the top 10 antisense RNAs significantly clustered patients with cancer and healthy individuals separately. Patients with stage I or II CRC exhibited significant changes in the expression levels of 33 sense and 39 antisense RNA species, as compared with healthy volunteers $(\mathrm{P}<0.01$; fold-change $>2.0)$. Cluster analysis demonstrated that patients with stage I or II CRC and healthy volunteers formed separate clusters only among the top 20 antisense RNA species. A tracking study of expression levels of haloacid dehalogenase-like hydrolase domain-containing 1 (HDHD1) antisense RNA was performed and a significant difference was identified between the CRC and healthy
\end{abstract}

Correspondence to: Professor Nobuhiro Ohkohchi, Department of Gastrointestinal and Hepato-Biliary-Pancreatic Surgery, Faculty of Medicine, University of Tsukuba, 1-1-1 Tennoudai, Tsukuba, Ibaraki 305-8575, Japan

E-mail: nokochi3@md.tsukuba.ac.jp

Key words: colorectal cancer, antisense RNA, haloacid dehalogenase-like hydrolase domain-containing 1, microarray groups revealing that the levels at one week and three months following surgical removal of the cancerous tissue, decreased to almost same levels of the healthy individuals. The results of the current study indicate that HDHD1 antisense RNA may serve as a potential biomarker for the prognosis of CRC.

\section{Introduction}

According to the American Cancer Society, colorectal cancer (CRC) is currently the third most malignant cancer in the United States among males and females (1). In the EU, CRC is the second most common cause of cancer-associated mortality in males, following lung cancer, and the third most frequent cause of cancer-associated mortality in females, following breast and lung cancer (2). In Japan, the number of patients with CRC has doubled in the past 20 years to become the second-leading cause of cancer-associated mortality (3).

CRC is a heterogeneous disease that occurs via a complex series of molecular events; a number of genes have previously been demonstrated to have a role in the development of the healthy mucosa of the large intestine into a benign tumor, which then transforms into an invasive cancer (4).

Previous comprehensive studies of $>13,000$ genes have identified 69 genes that are associated with the pathogenesis of CRC (4), and detailed analysis has revealed that an average of 9 mutant genes are involved in each case of CRC (5). Early $\mathrm{CRC}$ detection and the monitoring of patient prognosis are crucial to improve the survival rates of patients with CRC. However, the specimens and array analysis methods reported in previous studies have been problematic for the early diagnosis and monitoring of prognosis, and are not yet suitable for clinical use (4-6).

The development of microarray technology has facilitated the high-throughput analysis of numerous gene expression patterns $(5,6)$. CRC-specific gene expression profiles have been identified in mRNA (5,7-10). Furthermore, the analysis of non-coding RNA, including microRNA (miRNA) and antisense RNA, which cannot serve as templates for direct protein synthesis, has revealed associations between non-coding RNAs and the occurrence of certain types of cancer (11-13). Our previous studies demonstrated the potential involvement 
of antisense RNA expression profiles in the development of $\mathrm{CRC}$ and hepatic cancer by examining the expression patterns of specific RNAs in cancerous and healthy tissues $(14,15)$.

Blood samples are ideal for detection of certain types of cancer $(12,13)$ and the monitoring of prognosis; therefore, the present study evaluated RNA expression levels in the blood cells of patients, in order to determine their effectiveness in distinguishing cancerous states and healthy states. The current study identified that certain antisense RNA species in blood cells cluster separately in patients with CRC and healthy volunteers, and revealed that one of these RNA species may serve as a biomarker for prognosis.

\section{Materials and methods}

Patients and blood samples. Blood samples were collected from 6 healthy volunteers and from 28 patients with CRC who underwent surgical resection between April 2006 and March 2009 at Tsukuba University Hospital (Tsukuba, Japan). Blood sampling was periodically conducted up until 12 weeks after surgical resection. None of the patients had received radiotherapy or chemotherapy prior to surgery. The primary clinical characteristics for each patient with CRC involved in the current study are presented in Table I. Informed consent was obtained from all patients for the collection of blood samples and the ethics committee of Tsukuba University Hospital approved the study protocol.

Total RNA extraction from blood cells. Blood samples were collected from the patients using PAXgene Vacutainer tubes (BD Biosciences, Franklin Lakes, NJ, USA) and subjected to RNA isolation and extraction using a PAXgene Blood RNA Isolation kit (Qiagen, Inc., Valencia, CA, USA), following the manufacturer's instructions. The quantity of total RNA obtained was determined using a NanoDrop Spectrophotometer (NanoDrop Technologies; Thermo Fisher Scientific, Inc., Waltham, MA, USA) according to the 280/260 nm absorbance ratio, and RNA integrity was evaluated using an RNA 6000 Nano LabChip kit (Agilent Technologies, Inc., Santa Clara, CA, USA) and an Agilent 2100 Bioanalyzer (Agilent Technologies, Inc.).

Microarray analysis. Cyanine 3 (Cy3)-labeled cDNA was synthesized from $10 \mu \mathrm{g}$ total RNA extracted from the blood samples using a LabelStar Array kit (Qiagen, Inc.) with Cy3-dUTP (GE Healthcare Bio-Sciences, Pittsburgh, PA, USA) and random nonamer primers. The labeled cDNA was hybridized with probe sequences on an Agilent $44 \mathrm{Kx} 4$ human sense and antisense custom microarray slide (Agilent Technologies, Inc.) (16) in a hybridization solution prepared with an In Situ Hybridization Plus kit (Agilent Technologies, Inc.), according to the manufacturer's instructions. The $\mathrm{Cy} 3$ fluorescence signals were imaged using an Agilent C DNA microarray scanner (Agilent Technologies, Inc.) and processed using the Feature Extraction version 8.1 software (Agilent Technologies, Inc.).

Statistical analysis. The microarray data were processed using the GeneSpring GX version 12 software (Agilent Technologies, Inc.) to perform the log transformations and the normalization
Table I. Clinical characteristics of patients with colorectal cancer evaluated using microarray analysis $(\mathrm{n}=28)$.

\begin{tabular}{lc}
\hline Variable & Value \\
\hline Gender, $\mathrm{n}$ & \\
Male & 21 \\
Female & 7 \\
Median age (range), years & $60(37-86)$ \\
Tumor location, $\mathrm{n}$ & \\
Colon & 14 \\
Rectum & 14 \\
AJCC stage, $\mathrm{n}$ & \\
I & 9 \\
II & 9 \\
III & 6 \\
IV & 4 \\
Other samples, n & \\
1 week post-surgery samples & 4 \\
3 months post-surgery samples & 8 \\
Volunteers (non-cancer patients) & 6 \\
\hline
\end{tabular}

AJCC, American Joint Committee on Cancer.

of all values to the 75th percentile of the respective microarray, followed by normalization to the respective median expression level for all samples. Additionally, the normalized gene expression data were filtered on flags following the protocol of the manufacturer GeneSpring GX (http://www.chem.agilent.com/ cag/bsp/products/gsgx/manuals/GeneSpring-manual.pdf).

Only those genes classified as flag-present or flag-marginal in $>70 \%$ of all the array samples, were allowed to pass the filter. The expression profiles of the RNA samples were analysed, using GeneSpring GX version 12 software, with unpaired t-tests, with Benjamini-Hochberg false discovery rate correction (17) for unequal variances, as described in the results section. $\mathrm{P}<0.05$ was considered to indicate a statistically significant difference.

Two-dimensional hierarchical clustering based on Euclidean distance measures was performed using Ward's method (18). The data were visualized using heat maps and dendrograms, as described previously (14). Sample trees were drawn horizontally and gene trees were drawn vertically. Principal component analysis (PCA) was used to identify and characterize trends in multigene expression profiles.

\section{Results}

RNA quality. The quality of the RNAs obtained from the blood samples of the healthy volunteers and patients with CRC were initially examined using a NanoDrop Spectrophotometer at an absorbance ratio of $280 / 260 \mathrm{~nm}$, revealing that the ratios were between 1.8 and 2.0. These results indicated that the total RNAs prepared were usable for labeling with Cy3-dUTP and subsequent microarray analysis. The integrity of the RNAs was then examined using an Agilent 2100 Bioanalyzer, 
Table II. Top 20 antisense transcripts differentially regulated between blood cells from patients with colorectal cancer and healthy volunteers.

\begin{tabular}{lllc}
\hline Accession number & Gene symbol & \multicolumn{1}{c}{ Gene name } & Fold-change \\
\hline NM_012080 & HDHD1 & Haloacid dehalogenase-like hydrolase domain-containing 1 & 5.68 \\
NM_005824 & LRRC17 & Leucine-rich repeat containing 17 & 4.38 \\
XR_016125 & LOC642337 & Similar to hCG1648021 & 3.40 \\
NM_016630 & SPG21 & Spastic paraplegia 21 & 2.71 \\
XM_001132492 & LOC732276 & Hypothetical protein LOC732276 & 2.54 \\
NM_175611 & GRIK1 & Glutamate receptor, ionotropic, kainate 1 & 2.54 \\
NM_003290 & TPM4 & Tropomyosin 4 & 2.40 \\
NM_006516 & SLC2A1 & Solute carrier family 2, member 1 & 2.39 \\
NM_015317 & PUM2 & Pumilio homolog 2 & 2.36 \\
NM_024494 & WNT2B & Wingless-type MMTV integration site family, member 2B & -2.35 \\
NM_025140 & CCDC92 & Coiled-coil domain-containing 92 & 2.31 \\
NM_001037738 & NPM1 & Nucleophosmin 1 & 2.31 \\
NM_024860 & SETD6 & SET domain-containing 6 & 2.27 \\
NM_020179 & SMCO4 & Homo sapiens chromosome 11 open reading frame 75 (C11orf75) & 2.25 \\
XR_016982 & LOC645280 & Hypothetical LOC645280 & 2.21 \\
NM_025225 & PNPLA3 & Homo sapiens patatin-like phospholipase domain-containing 3 & 2.20 \\
NM_021975 & RELA & Homo sapiens v-rel avian reticuloendotheliosis viral oncogene homolog A & 2.03 \\
XM_926307 & LOC642927 & Similar to COLlagen family member (col-36) & 2.02 \\
NM_172249 & CSF2RA & Homo sapiens colony stimulating factor 2 receptor, alpha, low-affinity & 2.02 \\
NM_005206 & CPK & Homo sapiens v-crk avian sarcoma virus CT10 oncogene homolog & 2.00 \\
\hline
\end{tabular}

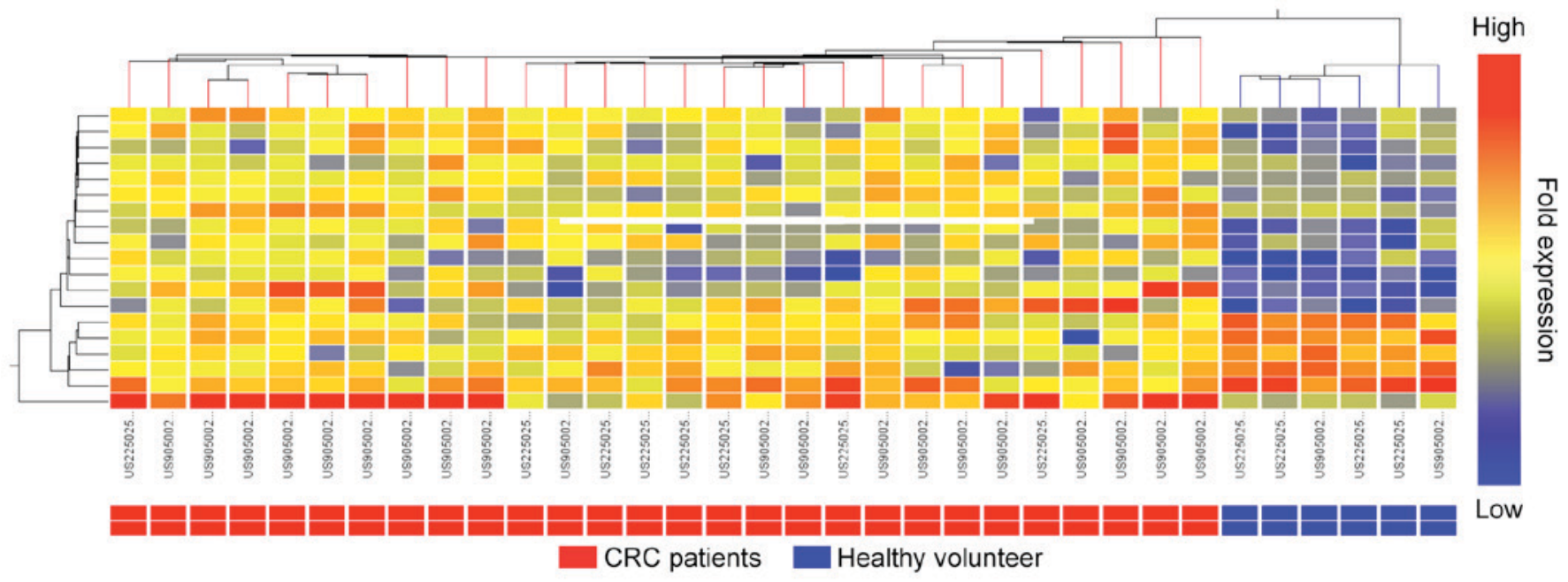

Figure 1. Hierarchical cluster analysis of the patients with CRC and the healthy volunteers using the top 20 antisense transcripts presented in Table II. Heat maps depict color-coded expression levels (the color gradation from red to blue indicates high to low expression levels). CRC, colorectal cancer.

revealing that the Rin values of the RNA samples ranged from 5.9 to 9.2 . As the random priming method was utilized for the synthesis of Cy3-labeled cDNAs from the RNA samples, the lower Rin values were considered not to affect the quality of the cDNAs for analysis.

Microarray analysis. Cy3-labeled cDNAs were synthesized from the extracted RNA and subjected to microarray analysis. A total of 40 transcripts were identified as differentially expressed with a magnitude of $>2$-fold $(\mathrm{P}<0.05)$ between the CRC blood cells and non-cancerous blood cells; of these transcripts, 20 were sense sequences and 20 were antisense sequences (Table II). According to the cluster analysis, CRC and non-cancerous blood samples were revealed to form separate clusters for the antisense transcripts, but not to form separate clusters for the sense transcripts (Fig. 1; the clustering data for the sense transcripts are not presented). 
Table III. Top 39 antisense transcripts differentially regulated between blood cells from patients with stage I/II colorectal cancer and healthy volunteers.

\begin{tabular}{|c|c|c|c|}
\hline Accession number & Gene symbol & Gene name & Fold-change \\
\hline XM_001132487.1ea & LOC732271 & Hypothetical protein LOC732271 & 6.24 \\
\hline NM_005824.1ea & LRRC17 & Leucine-rich repeat-containing 17 & 4.50 \\
\hline NM_012080.3ea & HDHD1 & Haloacid dehalogenase-like hydrolase domain-containing 1 & 3.57 \\
\hline NM_024422.2ia & $\mathrm{DSC} 2$ & Desmocollin 2 & 2.94 \\
\hline NM_174913.1ia & NOP9 & NOP9 nucleolar protein & 2.92 \\
\hline NM_016630.3ia & SPG21 & Spastic paraplegia 21 & 2.71 \\
\hline NM_024494.1ia & WNT2B & Wingless-type MMTV integration site family, member 2B & 2.61 \\
\hline NM_175611.2ea & GRIK1 & Glutamate receptor, ionotropic, kainate 1 & 2.54 \\
\hline NM_014578.2ia & RHOD & Ras homolog family member $\mathrm{D}$ & 2.54 \\
\hline NM_006296.3ia & VRK2 & Vaccinia-related kinase 2 & 2.47 \\
\hline NM_139284.1ia & LGI4 & Leucine-rich repeat LGI family & 2.44 \\
\hline NM_001315.1ia & MAPK14 & Mitogen-activated protein kinase 14 & 2.40 \\
\hline XR_016125.1ea & LOC642337 & Similar to hCG1648021 & 2.39 \\
\hline NM_005618.2ia & DLL1 & Felta-like 1 (Drosophila) & 2.37 \\
\hline XM_001132492.1ea & LOC732276 & Hypothetical protein LOC732276 & 2.36 \\
\hline NM_025225.2ia & PNPLA3 & Patatin-like phospholipase domain-containing 3 & 2.31 \\
\hline NM_171846.1ea & LACTB & Lactamase, $\beta$ & 2.31 \\
\hline NR_002162.1ea & ATP5EP2 & $\begin{array}{l}\text { Synthase, } \mathrm{H}+\text { transporting, mitochondrial F1 complex, epsilon } \\
\text { subunit pseudogene } 2\end{array}$ & 2.29 \\
\hline XM_001125928.1ea & LOC283804 & $\begin{array}{l}\text { Similar to testicular metalloprotease-like, disintegrin-like, } \\
\text { Cysteine-rich protein Iva }\end{array}$ & 2.25 \\
\hline XM_001129971.1ea & LOC729333 & Hypothetical protein LOC729333 & 2.23 \\
\hline NM_212554.2ia & METTL10 & Methyltransferase-like 10 & 2.22 \\
\hline NM_006516.1ia & SLC2A1 & Solute carrier family 2 , member 1 & 2.20 \\
\hline NM_015317.1ia & PUM2 & Pumilio homolog 2 & 2.18 \\
\hline XM_293121.4ea & C20orf66 & Chromosome 20 open reading frame 66 & 2.15 \\
\hline NM_003290.1ia & TPM4 & Tropomyosin 4 & 2.12 \\
\hline XR_016982.1ea & LOC645280 & Hypothetical LOC645280 & 2.12 \\
\hline XR_018204.1ea & LOC647757 & Similar to tetratricopeptide repeat protein 4 (TPR repeat protein 4$)$ & 2.09 \\
\hline NM_006708.1ia & GLO1 & Glyoxalase I & 2.08 \\
\hline NM_020179.1ia & SMCO4 & Single-pass membrane protein with coiled-coil domains 4 & 2.06 \\
\hline NM_001010898.1ia & SLC6A17 & Solute carrier family 6 (neutral amino acid transporter), member 17 & 2.06 \\
\hline NM_004423.3ia & DVL3 & Dishevelled segment polarity protein 3 & 2.05 \\
\hline NM_001093.2ia & $\mathrm{ACACB}$ & Acetyl-CoA carboxylase $\beta$ & 2.04 \\
\hline NM_005751.3ia & AKAP9 & A kinase (PRKA) anchor protein 9 & 2.04 \\
\hline NM_025140.1ia & CCDC92 & Coiled-coil domain-containing 92 & 2.04 \\
\hline NM_017440.2ia & MDM1 & Mdm1 nuclear protein homolog (mouse) & 2.04 \\
\hline NM_000637.2ia & GSR & Glutathione reductase & 2.03 \\
\hline NM_080588.1ia & PTPN7 & Protein tyrosine phosphatase, non-receptor type 7 & 2.02 \\
\hline NM_024860.1ia & SETD6 & SET domain-containing 6 & 2.01 \\
\hline NM_005206.3ia & CRK & V-crk avian sarcoma virus CT10 oncogene homolog & 2.01 \\
\hline
\end{tabular}

PCA for 28 patients with CRC using 20 antisense transcripts. As presented in Fig. 2, PCA analysis revealed that the CRC and non-cancerous blood samples were well separated with the first principal component; its contribution rate was $\sim 43.5 \%$. The contribution rates of the second and third principal components were calculated to be $\sim 7.7 \%$ and $\sim 6.4 \%$, respectively. These results indicate that the $\mathrm{CRC}$ and non-cancerous blood samples were effectively separated with only the first component.

Hierarchical cluster analysis of patients with stage I or II $C R C$. The results of the microarray with RNA samples from patients with stage I and II CRC and controls revealed a total of 72 transcripts that were significantly differentially expressed 


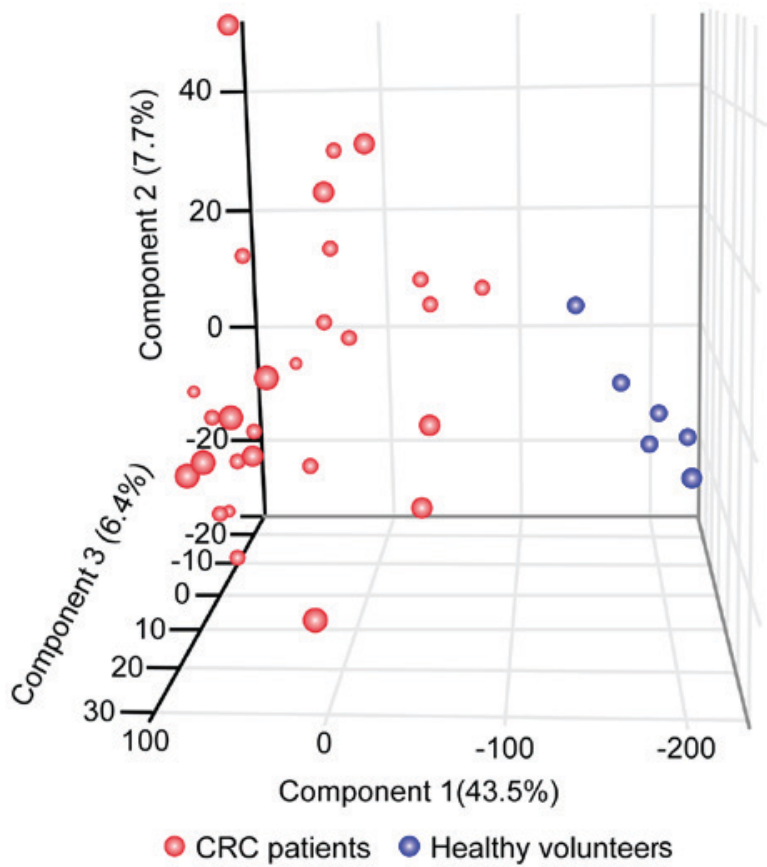

Figure 2. Principal component analysis for 28 patients with CRC and 6 healthy volunteers using the 20 antisense transcripts presented in Table II. The data are representative of the top three components; the contribution ratios of components 1,2 and 3 were $43.5,7.7$ and $6.4 \%$, respectively. CRC, colorectal cancer.

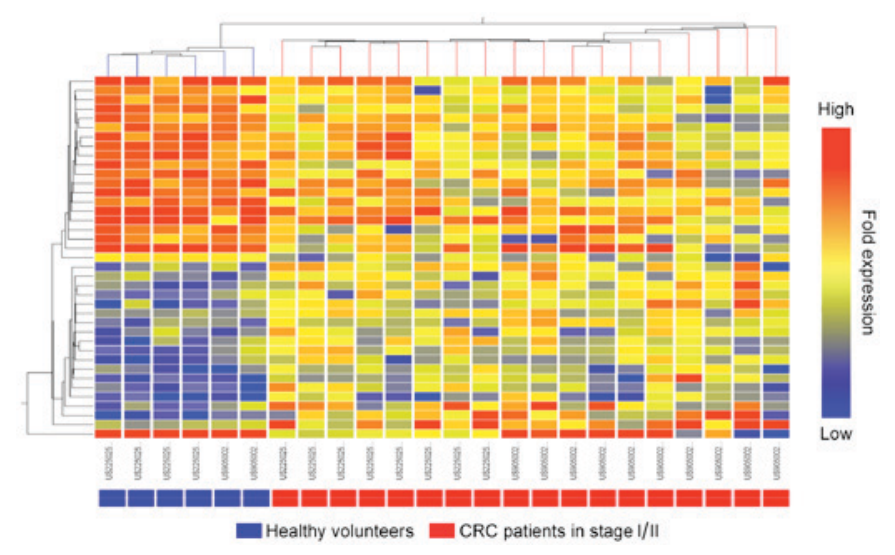

Figure 3. Hierarchical cluster analysis of patients with stage I or II CRC and healthy volunteers, using the top 39 antisense transcripts presented in Table III. Heat maps depict color-coded expression levels (the color gradation from red to blue indicates high to low expression levels). CRC, colorectal cancer.

with a magnitude of $>2$-fold $(\mathrm{P}<0.01)$ between the blood cells of healthy volunteers and of patients with CRC. A total of 33 transcripts were sense sequences and 39 were antisense sequences (Table III). When the blood samples from patients with stage I or II CRC and healthy volunteers were subjected to cluster analysis for the aforementioned sense and antisense transcripts, the patients were observed to form respective clusters only with antisense transcripts (Fig. 3; the clustering data for the sense transcripts are not presented).

PCA with stage I or II CRC using 39 antisense transcripts. The 39 antisense transcripts were further examined using PCA

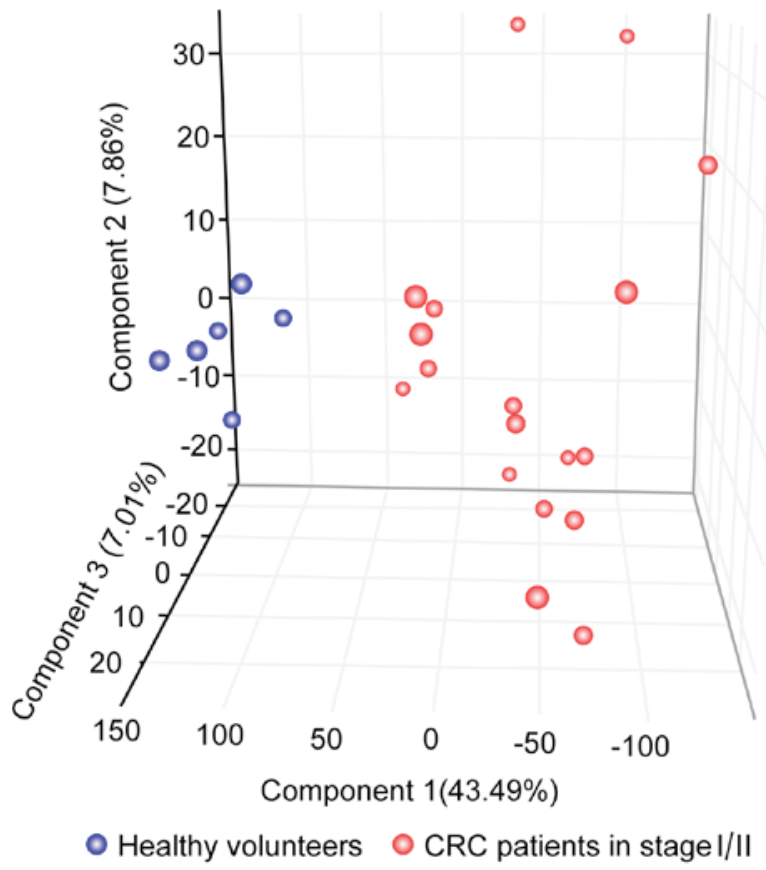

Figure 4. Principal component analysis for 18 patients with stage I or II CRC and 6 healthy volunteers using the 39 antisense transcripts presented in Table III. The data are representative of the top 3 components; the contribution ratios of components 1,2 and 3 were 43.5, 7.9 and $7.0 \%$, respectively. $\mathrm{CRC}$, colorectal cancer.

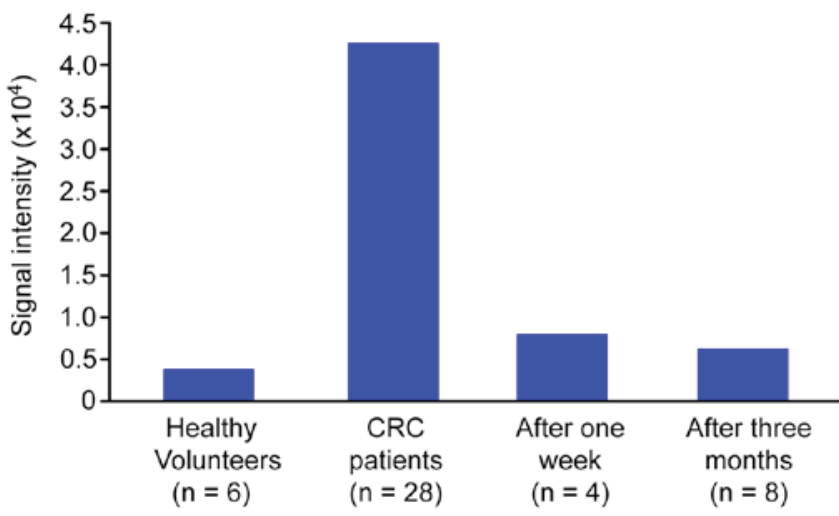

Figure 5. Comparison of haloacid dehalogenase-like hydrolase domain-containing 1 antisense transcript expression levels in the blood cell samples of healthy volunteers and patients with CRC prior to surgery and at one week and three months after surgery. CRC, colorectal cancer.

analysis. As presented in Fig. 4, CRC and non-cancerous RNA samples were revealed to be well separated with regard to the first principal component; its contribution rate was $\sim 43.5 \%$. The contribution rates of the second and third principal components were calculated to be $\sim 7.9 \%$ and $\sim 7.0 \%$, respectively. These results indicate that the patients with stage I or II CRC were effectively separated from healthy volunteers with the first component.

Comparison of HDHDI antisense transcript expression levels. The antisense transcript of haloacid dehalogenase-like hydrolase 1 domain-containing (HDHD1), which was the highest and third highest differentially expressed transcript in the earlier analyses (Tables II and III, respectively), was 
selected for evaluation at various time points following the surgical resection of tumor tissues from patients with CRC. The expression levels of the HDHD1 antisense transcript at 1 week and at 3 months post-surgery were decreased approximately to the expression levels observed in healthy volunteers (Fig. 5). The results suggest that certain antisense transcripts, including HDHD1, may serve as potential biomarkers of CRC diagnosis and prognosis.

\section{Discussion}

Previous studies have identified the potential involvement of antisense RNA expression in the development of colorectal and hepatic cancers by examining RNA expression patterns in cancerous and healthy tissues $(14,15)$. However, the functions and underlying mechanisms of antisense transcripts in colorectal and hepatic cancers have yet to be elucidated. The present study examined the association between the expression levels of certain RNA transcripts in blood cells and the occurrence of CRC, and the subsequent changes in the transcript amount following the removal of cancerous tissues in patients. The examinations revealed that antisense transcripts of up to 39 genes demonstrated a clear association between their expression levels in blood cells and the occurrence of CRC; following tumor resection in patients with CRC, the expression levels of the HDHD1 antisense transcript were decreased to approximately the levels observed in healthy volunteers, suggesting that these antisense transcripts are involved in the generation and maintenance of CRC. Furthermore, the antisense transcripts may serve as diagnostic markers for CRC occurrence, and certain antisense transcripts, including HDHD1, may be potential prognostic markers for CRC.

The early detection of CRC significantly improves patient prognosis and is essential in reducing CRC-associated mortality (19) Patients with CRC often present with an advanced stage disease and concomitant poor prognosis (1). The best known serum biomarkers, carcinoembryonic antigen (CEA) and carbohydrate antigen 19-9 (CA19-9), are not recommended for clinical screening due to limited specificity and sensitivity (20). A number of circulating proteins have previously been indicated to be diagnostically useful; however, none of these proteins has individually demonstrated sufficient sensitivity or specificity to be used in clinical practice (20).

In our previous study, the expression levels of certain antisense transcripts in CRC tissues were revealed to significantly differ from their corresponding normal tissues (14), indicating that those specific antisense transcripts may be involved in the generation and maintenance of CRC tumor tissues. The antisense transcripts in CRC tissues are distinct from those identified in the present study of patients with CRC. Further studies are required to determine the functional association between the antisense transcripts revealed in our previous study and those in the present study.

Previous studies have indicated that certain antisense transcripts are involved in mRNA stabilization (21), the suppression of mRNA synthesis $(22,23)$, miRNA functions (24) and the promotion of protein synthesis (25).
Therefore, antisense transcripts have various functions, each of which may be specific to the respective antisense transcript species. Although the functions of non-coding RNA, antisense RNA and miRNA in tumorigenesis require further study, it is possible that the various antisense transcripts demonstrated in the present study may serve as potential biomarkers for $\mathrm{CRC}$ diagnosis and prognosis. In the future, the mechanisms underlying the differences in expression levels of the antisense transcripts should be investigated extensively to understand their involvement in CRC generation.

\section{Acknowledgements}

This study was supported in part by the Ministry of Education, Culture, Sports, Science and Technology of Japan.

\section{References}

1. Jemal A, Siegel R, Xu J and Ward E: Cancer Statistics, 2010. CA Cancer J Clin 60: 277-300, 2010.

2. Malvezzi M, Bertuccio P, Levi F, La Vecchia C and Negri E: European cancer mortality predictions for the year 2014. Ann Oncol 25: 1650-1656, 2014.

3. Tsukuma H, Ajiki W and Oshima A: Cancer incidence in Japan. Gan To Kagaku Ryoho 31: 840-846, 2004 (In Japanese).

4. Koehler A, Bataille F, Schmid C, Ruemmele P, Waldeck A, Blaszyk H, Hartmann A, Hofstaedter F and Dietmaier W: Gene expression profiling of colorectal cancer and metastases divides tumours according to their clinicopathological stage. J Pathol 204: 65-74, 2004

5. Croner RS, Foertsch T, Brueckl WM, Guenther K, Siebenhaar R, Stremmel C, Matzel KE, Papadopoulos T, Kirchner T, Behrens J, et al: Common denominator genes that distinguish colorectal carcinoma from normal mucosa. Int J Colorectal Dis 20: 353-362, 2005.

6. Raetz EA and Moos PJ: Impact of microarray technology in clinical oncology. Cancer Invest 22: 312-320, 2004.

7. Ohmachi T, Tanaka F, Mimori K, Inoue H, Yanaga K and Mori M: Clinical significance of TROP2 expression in colorectal cancer. Clin Cancer Res 12: 3057-3063, 2006.

8. Bertucci F, Salas S, Eysteries S, Nasser V, Finetti P, Ginestier C, Charafe-Jauffret E, Loriod B, Bachelart L, Montfort J, et al: Gene expression profiling of colon cancer by DNA microarrays and correlation with histoclinical parameters. Oncogene 23: 1377-1391, 2004.

9. Bianchini M, Levy E, Zucchini C, Pinski V, Macagno C, De Sanctis P, Valvassori L, Carinci P and Mordoh J: Comparative study of gene expression by cDNA microarray in human colorectal cancer tissues and normal mucosa. Int J Oncol 29: 83-94, 2006.

10. Birkenkamp-Demtroder K, Christensen LL, Olesen SH, Frederiksen CM, Laiho P, Aaltonen LA, Laurberg S, Sørensen FB, Hagemann R and ØRntoft TF: Gene expression in colorectal cancer. Cancer Res 62: 4352-4363, 2002.

11. Lawlor KG, Telang NT, Osborne MP, Schaapveld RQ, Cho KR, Vogelstein B and Narayanan R: Antisense RNA to the putative tumor suppressor gene ‘deleted in colorectal cancer' transforms fibroblasts. Ann N Y Acad Sci 660: 283-285, 1992.

12. Chen X, Ba Y, Ma L, Cai X, Yin Y, Wang K, Guo J, Zhang Y, Chen J, Guo X, et al: Characterization of microRNAs in serum: A novel class of biomarkers for diagnosis of cancer and other diseases. Cell Res 18: 997-1006, 2008.

13. Grigoriadis A, Oliver GR, Tanney A, Kendrick H, Smalley MJ, Jat P and Neville AM: Identification of differentially expressed sense and antisense transcript pairs in breast epithelial tissues. BMC Genomics 10: 324, 2009.

14. Kohno K, Chiba M, Murata S, Pak S, Nagai K, Yamamoto M, Yanagisawa K, Kobayashi A, Yasue H and Ohkohchi N: Identification of natural antisense transcripts involved in human colorectal cancer development. Int J Oncol 37: 1425-1432, 2010.

15. Nagai K, Kohno K, Chiba M, Pak S, Murata S, Fukunaga K, Kobayashi A, Yasue H and Ohkohchi N: Differential expression profiles of sense and antisense transcripts between HCV-associated hepatocellular carcinoma and corresponding non-cancerous liver tissue. Int J Oncol 40: 1813-1820, 2012. 
16. Chiba M, Kimura M and Asari S: Exosomes secreted from human colorectal cancer cell lines contain mRNAs, microRNAs and natural antisense RNAs, that can transfer into the human hepatoma HepG2 and lung cancer A549 cell lines. Oncol Rep 28: 1551-1558, 2012.

17. Benjamini $\mathrm{Y}$ and Hochberg Y: Controling the false discovery rate: A pratical and powerful approach to multiple testing. J R Statist Soc 57: 289-300, 1995.

18. Ward JH Jr: Hierarchical grouping to optimize an objective function. J Am Stat Assoc 58: 236-244, 1963.

19. Etzioni R, Urban N, Ramsey S, McIntosh M, Schwartz S, Reid B, Radich J, Anderson G and Hartwell L: The case for early detection. Nat Rev Cancer 3: 243-252, 2003.

20. Hundt S, Haug U and Brenner H: Blood markers for early detection of colorectal cancer: A systematic review. Cancer Epidemio Biomarkers Prev 16: 1935-1953, 2007.

21. Yoshigai E, Hara T, Araki Y, Tanaka Y, Oishi M, Tokuhara K, Kaibori M, Okumura T, Kwon AH and Nishizawa M: Natural antisense transcript-targeted regulation of inducible nitric oxide synthase mRNA levels. Nitric Oxide 30: 9-16, 2013.
22. Robb GB, Carson AR, Tai SC, Fish JE, Singh S, Yamada T, Scherer SW, Nakabayashi K and Marsden PA: Post-transcriptional regulation of endothelial nitric-oxide synthase by an overlapping antisense mRNA transcript. J Biol Chem 279: 37982-37996, 2004.

23. Camblong J, Iglesias N, Fickentscher C, Dieppois G and Stutz F: Antisense RNA stabilization induces transcriptional gene silencing via histone deacetylation in S. Cerevisiae. Cell 131: 706-717, 2007.

24. Faghihi MA, Żhang M, Huang J, Modarresi F, Van der Brug MP Nalls MA, Cookson MR, St-Laurent G III and Wahlestedt C: Evidence for natural antisense transcript-mediated inhibition of microRNA function. Genome Biol 11: R56, 2010.

25. Carrieri C, Cimatti L, Biagioli M, Beugnet A, Zucchelli S, Fedele S, Pesce E, Ferrer I, Collavin L, Santoro C, et al: Long non-coding antisense RNA controls Uchl1 translation through an embedded SINEB2 repeat. Nature 491: 454-457, 2012. 